Check for updates

Cite this: RSC Adv., 2017, 7, 19288

\title{
Non-swellable self-healing polymer with long-term stability under seawater $\dagger$
}

\begin{abstract}
Chaehoon Kim, ${ }^{a}$ Hirotaka Ejima ${ }^{\mathrm{b}}$ and Naoko Yoshie (iD *a
Polymers are widely used in marine environments due to their unique thermal/mechanical properties. Imparting self-healing ability to those polymers would be a beneficial strategy for improved durability; e.g. extending the life time and reducing the maintenance cost. However, most of the existing self-healing polymers suffer from swelling-induced mechanical instability and loss of self-healing ability because of a severe water uptake in fully submerged conditions. Thus, a judicious design principle to prevent the swelling-induced deterioration of the self-healing polymers is greatly required for the long-term use in water-related applications. In this study, we report a polymer that is non-swellable ( $<2 \mathrm{wt} \%$ of swelling) and highly self-healable $(91 \%$ based on toughness) under seawater. Dynamic crosslinking of catecholfunctionalized polymers with $p$-phenyldiboronic acid (PDBA) through non-ionic boronate ester bonds is the key to realizing these two properties simultaneously. Atomic force microscopy and transmission electron microscopy reveal that the ionically crosslinked catechol polymers contain hydrophilic aggregates of metal $\left(\mathrm{Ca}^{2+}\right)$-catechol complexes, referred to as ion-clusters. In contrast, we find the non-ionic boronate ester crosslinkers are not aggregated but finely dispersed in hydrophobic catechol polymer matrix, allowing materials to self-heal with high water stability. Even in seawater, the PDBA-containing catechol polymer retained its mechanical properties for at least 1 month. The polymer described here could provide a valuable strategy for further development of polymeric materials used in marine industries.
\end{abstract}

Received 13th February 2017
Accepted 22nd March 2017

DOI: $10.1039 / c 7 r a 01778 b$

rsc.li/rsc-advances in dry conditions has been extensively studied for polymers with reversible bonds. ${ }^{5-10}$ Recently, self-healing in wet conditions was also achieved in a few reports in which the water triggered dynamics of a hydrogen bond interaction, ${ }^{\mathbf{1 1}}$ a boronic ester bond ${ }^{\mathbf{1 2}}$ and metal-ligand co-ordinations ${ }^{\mathbf{1 3 , 1 4}}$ were used to facilitate the repair. Ahn and co-workers proved that the hydrogen bonding interaction can be used in self-healable bulk polymers under wet conditions. ${ }^{\mathbf{1 1}}$ By deprotection of silyl-protecting groups in acidic condition, the newly generated hydrogen bonds between catechol groups led cracked surfaces to heal. Cash and co-workers ${ }^{\mathbf{1 2}}$ reported that a boronic ester bond was used as a dynamic crosslinker within hydrophobic network polymers, and successful self-healing was achieved with water treatment at the fractured surfaces.

Despite these advances, accomplishing self-healing in fully submerged environments without any external stimuli is still challenging because water-assisted healing is usually accompanied by swelling. In the case of hydrophilic polymers, swelling with water enhances the polymer chain mobility, leading to a higher probability of reformation of dynamic bonds and to successful self-healing at cracked interfaces. However, this swelling also induces severe structural deformation ${ }^{15}$ and mechanical property changes, ${ }^{16}$ causing these materials to fail during underwater use. Recently, our group ${ }^{13}$ and Xia and coworkers $^{\mathbf{1 4}}$ investigated catechol polymers in the bulk state and revealed relatively high self-healing efficiencies triggered by 
seawater. Enhanced chain mobility by water absorption and the dynamics of the catechol-metal (i.e., $\mathrm{Ca}^{2+}, \mathrm{Mg}^{2+}$, and $\mathrm{Fe}^{3+}$ ) complexes of these polymers led to successful healing efficiency, suggesting that catechol-containing polymers can be used as tough, self-healing materials in wet conditions. Nevertheless, severe water uptake occurred when these materials were immersed in seawater $(\sim 20 \mathrm{wt} \%),{ }^{\mathbf{1 3}, 14}$ indicating that a significant challenge remains for their use under the sea.

Although hydrophobic polymers maintain their intrinsic properties in water without swelling, the formation of a water boundary layer at cracked surfaces drastically weakens the van der Waals interactions, which results in interruption of the reformation of the dynamic bonds. ${ }^{17}$ Therefore, a judicious polymer design with an appropriate hydrophobicity along with water-triggered dynamic bonds is crucial for obtaining underwater self-healing elastomers with high stability.

To overcome this challenge, we herein develop a highly selfhealable tough polymer which can be used under seawater. For achieving such properties, the effect of the electrical nature of dynamic bonds on the water stability of self-healing elastomers is considered. The catechol-based polymer was synthesized as a hydrophobic matrix, and two different crosslinkers were chosen; one is a metalloid (boron) and the other is a metal (calcium). The elastomer networked with the $p$-phenyldiboronic acid (PDBA) shows non-swellable ( $<2$ wt $\%$ water absorption) and self-healable ( $91 \%$ efficiency) properties under seawater with stable mechanical properties. Such healable properties in fully submerged conditions have never been achieved before. Contrary, the one networked with the $\mathrm{Ca}^{2+}$ suffered from the swelling-induced mechanical deterioration. We relate these phenomena to the mesoscopic structure differences revealed by atomic force microscopy (AFM) and transmission electron microscopy (TEM).

\section{Experimental section}

\section{Reagents and materials}

As solvents, we used methanol, dichloromethane, chloroform, acetone, hexane, ethyl acetate, $N, N$-dimethylformamide (DMF, dehydrated), pyridine (dehydrated), ethanol (dehydrated), and tetrahydrofuran (THF, dehydrated, stabilizer-free), all of which were obtained from Wako Pure Chemical Industries, Ltd. Acryloyl chloride, triethylamine (TEA), butyl acrylate, dimethyl 2,2'-azobis(2-methylpropionate), $p$-phenyldiboronic acid and calcium chloride were also obtained from Wako Pure Chemical Industries, Ltd. Acrylic acid was purchased from Sigma-Aldrich. Dopamine hydrochloride was purchased from Tokyo Chemical Industry Co., Ltd. Butyl acrylate was purified by passing it through basic alumina before use. The artificial seawater of which contents are listed in Table $\mathrm{S} 1 \uparrow$ was used for self-healing, swelling and long-term stability tests.

\section{Characterization}

${ }^{1} \mathrm{H}$ NMR spectra were recorded on a JNM-AL 400 (JEOL RESONANCE Inc.) in deuterated DMSO at $20{ }^{\circ} \mathrm{C}$. Solid-state ${ }^{11} \mathrm{~B}$ NMR spectra were recorded on an ECA-500 (JEOL RESONANCE Inc.) with a $4 \mathrm{~mm}$ zirconia rotor. The magic angle spinning speed was $16 \mathrm{kHz}$. The saturated $\mathrm{H}_{3} \mathrm{BO}_{3}$ aqueous solution (-19.49 ppm) served as an external chemical shift standard. Gel permeation chromatography (GPC) data were recorded on a HLC-8220 GPC (TOSOH Co.) using $10 \mathrm{mM} \mathrm{LiCl} \mathrm{in} \mathrm{DMF} \mathrm{as} \mathrm{the} \mathrm{eluent.} \mathrm{A} \mathrm{cali-}$ bration curve was prepared by using polystyrene standards. Attenuated total reflection-Fourier transform infrared (ATRFTIR) spectra were obtained with a Thermo Scientific Nicolet iS10 FTIR spectrometer equipped with a Thermo Scientific Smart iTR with a diamond crystal. Differential scanning calorimetry (DSC) data were measured using a SEIKO DSC6220N at a heating rate of $20{ }^{\circ} \mathrm{C} \mathrm{min}{ }^{-1}$ under nitrogen (flow rate $=5 \mathrm{~mL}$ $\left.\mathrm{min}^{-1}\right)$. The data were recorded from the second heating process $\left(-50{ }^{\circ} \mathrm{C}\right.$ to $\left.150{ }^{\circ} \mathrm{C}\right)$ after a single scan $\left(-50{ }^{\circ} \mathrm{C}\right.$ to $\left.80{ }^{\circ} \mathrm{C}\right)$. Tensile tests were conducted by SHIMADZU AGS-X $100 \mathrm{~N}$ and the dimensions of the dumbbell-shaped sample used are shown in Fig. S5. $\uparrow$ The strain rate of $30 \mathrm{~mm} \mathrm{m^{-1 }}$ was adopted under ambient condition $\left(25{ }^{\circ} \mathrm{C}, 60 \%\right.$ of relative humidity). Each measurement was repeated at least three times. Water contact angle measurements were operated by DMs-401 (Kyowa Interface Science Co., Ltd.). A droplet of deionized water $(1 \mu \mathrm{L})$ was used for each measurement with high-resolution video. The initial contact angle was measured at $100 \mathrm{~ms}$ after water dropping. Data are mean of more than 3 measurements. AFM measurements were operated by a Nanocute (SII Nano Technology, Inc.) with a self-sensitive micro-cantilever PRC-DF40P, using the dynamic force mode. TEM images were obtained with a JEM-2010 TEM (JEOL, Ltd.), operating at $200 \mathrm{kV}$. Selfhealing image and the surface roughness of films were observed using a laser scanning microscopy (VK-X210, Keyence).

\section{Preparation of catechol containing polymers}

The procedure was slightly modified from our previous report. ${ }^{13}$ To a nitrogen-purged $200 \mathrm{~mL}$ Schlenk flask, $7.46 \mathrm{~g}$ of dopamine acrylamide (DA, $36 \mathrm{mmol}), 410 \mathrm{mg}$ of dimethyl 2,2'-azobis(2methylpropionate) (1.8 mmol), $46.7 \mathrm{~mL}$ of $n$-butyl acrylate (BA, $324 \mathrm{mmol}$ ), and $150 \mathrm{~mL}$ of anhydrous ethanol was added. The solution was degassed via three freeze-pump-thaw cycles and backfilled with nitrogen. Then the solution was placed in an oil bath at $75{ }^{\circ} \mathrm{C}$ and stirred for $24 \mathrm{~h}$. The solution was quenched by liquid nitrogen and reduced in volume to $\sim 50 \mathrm{~mL}$ by rotary evaporation under reduced pressure. THF $(30 \mathrm{~mL})$ was then added, and the resulting solution was precipitated by pouring into $0{ }^{\circ} \mathrm{C}$ hexane. The precipitate was collected and dried under vacuum to yield a yellowish liquid-like product $(42.5 \mathrm{~g}$, yield 87\%). ${ }^{1} \mathrm{H}$ NMR (400 MHz, DMSO-d 6 , $\delta$ ): 2.17-0.88 (93H, aliphatic region), $2.47\left(2 \mathrm{H}, \mathrm{CONH}-\mathrm{CH}_{2}-\mathrm{CH}_{2}\right), 3.12\left(2 \mathrm{H}, \mathrm{CONH}-\mathrm{CH}_{2}-\right.$ $\mathrm{CH}_{2}$ ), $3.97\left(18 \mathrm{H}, \mathrm{COO}-\mathrm{CH}_{2}-\mathrm{CH}_{2}-\mathrm{CH}_{2}-\mathrm{CH}_{3}\right), 6.62-6.40(3 \mathrm{H}, \mathrm{Ar})$, $7.86(1 \mathrm{H}, \mathrm{NH}), 8.71(2 \mathrm{H}, \mathrm{ArOH})$. GPC $(10 \mathrm{mM} \mathrm{LiCl}$ in DMF as eluent, polystyrene standards): $M_{\mathrm{n}}=79000, M_{\mathrm{w}} / M_{\mathrm{n}}=1.9$.

\section{Preparation of network polymers}

In a typical film preparation of P-PDBA $100 \%$ and $\mathrm{P}^{-\mathrm{Ca}^{2+}} 100 \%$, $1.8 \mathrm{~g}$ of $\mathrm{P}(\mathrm{DA}-\mathrm{co}-\mathrm{BA})$ (containing $1.33 \mathrm{mmol}$ catechol) was dissolved in $60 \mathrm{~mL}$ of chloroform. Then $3 \mathrm{~mL}$ of a mixture of crosslinker in methanol (PDBA $110 \mathrm{mg}$ or $\mathrm{CaCl}_{2} 74 \mathrm{mg}$ ) and $0.185 \mathrm{~mL}$ 
of TEA (1.33 mmol) was added. The solution was stirred for $24 \mathrm{~h}$, poured into a Teflon dish, and allowed to evaporate for $24 \mathrm{~h}$ at room temperature. The sample was then put in a vacuum oven at $50{ }^{\circ} \mathrm{C}$ for another $24 \mathrm{~h}$ to remove the remaining solvent. A film with an average thickness of $0.3 \mathrm{~mm}$ was prepared by thermal annealing $\left(60{ }^{\circ} \mathrm{C}, 30 \mathrm{~min}\right)$ with compression between two sheets of Teflon (12.5 MPa).

\section{Sample preparation for AFM}

Glass slides $(18 \times 18 \mathrm{~mm})$ were first washed with deionized (DI) water under sonication and then immersed in Piranha solution (3/1 v/v mixture of $96 \%$ sulfuric acid and $30 \% \mathrm{H}_{2} \mathrm{O}_{2}$ ) for $1 \mathrm{~h}$ to remove organic impurities. (CAUTION: Piranha solution can react violently with organic compounds, and should be handled with extreme care.) The glass slides were rinsed with DI water, sonicated in DI water, and then dried in air. The casting solution for bulk film is diluted 3-fold with $\mathrm{CHCl}_{3}$ and spin-coated onto the washed glass slide at $2000 \mathrm{rpm}$ for $20 \mathrm{~s}$ and then dried in air for $2 \mathrm{~h}$, in vacuum chamber at room temperature for $2 \mathrm{~h}$ and annealed at $60{ }^{\circ} \mathrm{C}$ for $30 \mathrm{~min}$. The dried sample was kept inside the desiccator until AFM observation.

\section{Sample preparation for TEM}

The solution for bulk film is diluted 60 -fold with $\mathrm{CHCl}_{3}$ and drop-casted onto the carbon coated copper grid. After drying out the solvent under ambient air for $24 \mathrm{~h}$, sample was annealed at $60{ }^{\circ} \mathrm{C}$ for $30 \mathrm{~min}$. The dried sample was kept inside the vacuum chamber until TEM observation.

\section{Self-healing tests}

Dumbbell-shaped samples were cut into two separate sections, and exposed surfaces were activated with a drop of seawater then rejoined gently under air. Reattached samples were fully immersed in seawater. After the desired period of time (1-3 days), samples were taken out from seawater and dried under vacuum for $12 \mathrm{~h}$ and then in a desiccator for another $12 \mathrm{~h}$ to remove any remaining water. Before tensile tests, samples were placed in ambient air $\left(25{ }^{\circ} \mathrm{C}, 60 \%\right.$ of relative humidity) for at least $30 \mathrm{~min}$ then subjected to tensile tests.

\section{Swelling tests}

Square-shaped samples $(10 \times 10 \mathrm{~mm})$ were cut from films with a pair of scissors. The samples were immersed in artificial seawater or deionized water for 14 days. Before weighing, the samples were carefully wiped with absorbent paper. Swelling ratios were calculated according to the equation: $100 \times$ $\left(W_{\text {measured }}-W_{\text {initial }}\right) / W_{\text {initial }}$, where $W_{\text {measured }}$ is the measured weight and $W_{\text {initial }}$ is the initial weight.

\section{Results and discussion}

\section{Polymer design}

The catechol containing polymer was synthesized as a hydrophobic polymer chain, because catechol is an adaptable ligand which can chelate with various metals ${ }^{18-21}$ and metalloids. ${ }^{22}$ For this purpose, poly(dopamine acrylamide-co- $n$-butyl acrylate) [P(DA-co-BA $)]$ was prepared by a conventional free radical copolymerization of $n$-butyl acrylate (BA) and dopamine acrylamide (DA) (Scheme S1 $\dagger$ ). ${ }^{1} \mathrm{H}$ NMR revealed that the comonomer composition of $9: 1$ (BA : DA) in the resulting product, closely corresponded to the feed ratio of the monomers (Fig. S1†). For the preparation of the crosslinked polymer, the $\mathrm{P}(\mathrm{DA}-\mathrm{co}-\mathrm{BA})$ (1.8 g, containing $1.33 \mathrm{mmol}$ catechol) in chloroform $(60 \mathrm{~mL})$ was mixed with a PDBA $(110 \mathrm{mg}, 0.67 \mathrm{mmol})$ in methanol (3 mL). Triethylamine (TEA, $1.33 \mathrm{mmol}, 2$ equiv. of PDBA) was also added to this solution for the effective formation of non-ionic boronate ester bonds. ${ }^{23}$ This sample is abbreviated P-PDBA $100 \%$ based on a bidentate coordination type between catechol moieties in the polymer chain and PDBA. Compositions containing 80 and 60\% feed ratios of PDBA were also prepared. A polymer film was then prepared by solution casting on a Teflon dish followed by thermal annealing with compression to yield cross-linked bulk films with high transparency (Fig. 1a). A $\mathrm{P}\left(\mathrm{DA}-\right.$-co-BA) film crosslinked with $\mathrm{Ca}^{2+}$ was also prepared by adding $\mathrm{CaCl}_{2}$ in place of PDBA, as a control which forms typical ionic metal-ligand interactions in the polymer matrix (abbreviated $\mathrm{P}-\mathrm{Ca}^{2+} 100 \%$ ). Unlike the transparent P-PDBA $100 \%$, the $\mathrm{P}-\mathrm{Ca}^{2+} 100 \%$ film was opaque and dark brown in color (Fig. 1b). The $T_{\mathrm{g}}$ values of all the network polymers were below $-10{ }^{\circ} \mathrm{C}$, as determined by differential scanning calorimetry (DSC) measurements (Table 1).

\section{Network characterization}

Characterization using solid-state NMR. Formation of the boronate ester bonds in P-PDBA $100 \%$ was confirmed by solidstate single-pulse ${ }^{11} \mathrm{~B}$ NMR spectroscopy (Fig. 2a). A sharp signal was detected at $8.6 \mathrm{ppm}$, which supported the formation of the tetrahedral $\mathrm{sp}^{3}$ boron center by $\mathrm{B}-\mathrm{N}$ dative bond. ${ }^{24-26}$ When a mixture of $\mathrm{P}(\mathrm{DA}-\mathrm{co}-\mathrm{BA})$ and PDBA was prepared without TEA, a robust film was not formed (Fig. $\mathrm{S} 2 \dagger$ ), and only a broad signal at $24 \mathrm{ppm}$ was observed in the ${ }^{11} \mathrm{~B}$ NMR spectrum (Fig. 2a). These results indicate that most of the PDBA was only reacted in the presence of TEA to contribute to the crosslinking.

Characterization using attenuated total reflectance-Fourier transform-infrared spectrometry (ATR-FTIR). ATR-FTIR spectroscopy of polymers with varying feed ratios of PDBA $(60,80$ and $100 \%$ ) also proved coordination between the catechol and boronic acid moieties. The ATR-FTIR spectra in Fig. 2b shows that two $\delta(\mathrm{C}-\mathrm{OH})$ bands at 1243 and $1254 \mathrm{~cm}^{-1}$ resulting from the phenolic groups of catechol in $\mathrm{P}(\mathrm{DA}-\mathrm{co}-\mathrm{BA})$ were merged into a single peak at $1248 \mathrm{~cm}^{-1}$ in the P-PDBA samples as a result of esterification between the catechol and boronic acid groups. $^{27}$

\section{Mechanical properties of P-PDBA polymers}

The mechanical properties of the P-PDBA samples were determined by tensile tests and dynamic mechanical studies. Table 1 shows the tensile test results of the P-PDBA polymers. As the amount of added PDBA increased, higher Young's modulus and break strength values were obtained. Among the P-PDBAs, PPDBA $100 \%$ displayed the toughest mechanical properties 
a)

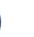

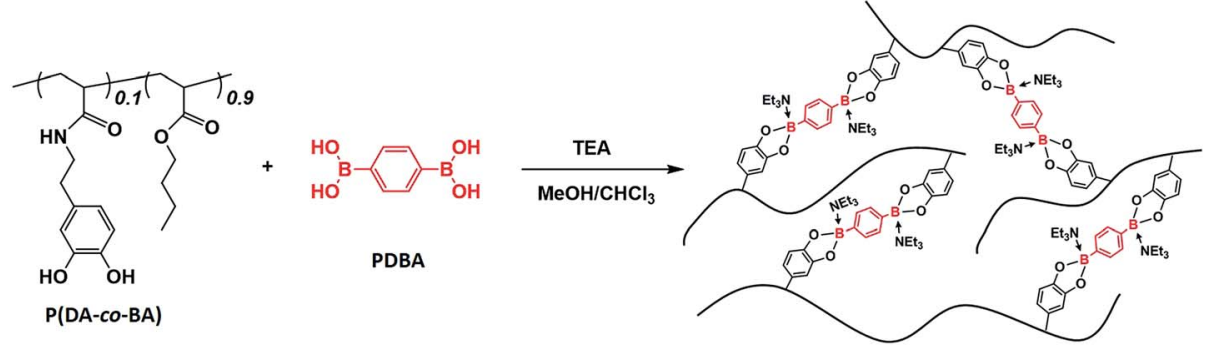

b)
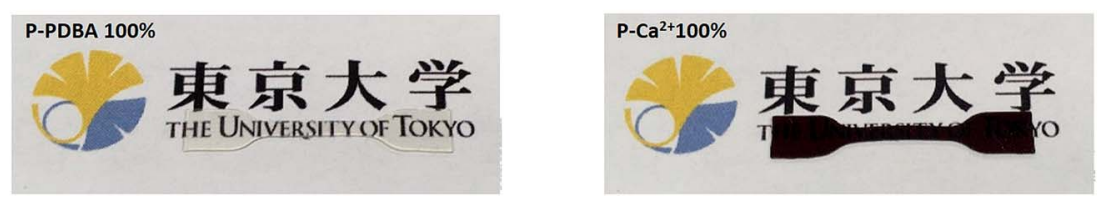

Fig. 1 (a) Synthesis of a catechol-containing polymer crosslinked with PDBA. (b) P-PDBA 100\% has high transparency (left), whereas P-Ca ${ }^{2+}$ $100 \%$ shows opaque and dark brown in color (right).

$\left(10.6 \mathrm{MJ} \mathrm{m}^{-3}\right.$ ), while P-PDBA $80 \%$ and $60 \%$ still exhibited fairly high toughness (10.0 and 9.2 $\mathrm{MJ} \mathrm{m}^{-3}$, respectively). In Fig. $\mathrm{S} 3, \dagger$ the heights of the loss tangent peaks, which relate to the relaxation of the polymer chain, were compared. By increasing the feed ratio of PDBA, the height of the peak decreased, suggesting that relaxation of the polymer chain was restricted to a larger degree by higher crosslinking densities.

\section{Self-healing properties of P-PDBA polymers}

The self-healing test in seawater was performed with P-PDBA $100 \%$. Because this polymer possessed the highest strength and the highest Young's modulus measured in this study, PPDBA $100 \%$ was considered to be the most challenging when it came to displaying self-healing properties. Dumbbell-shaped films were cut completely into two pieces, and the exposed surfaces were wetted with seawater before gently rejoining the two pieces. The rejoined films were then fully immersed in seawater for 1-3 days (Fig. 3a). After drying, tensile tests of the films were conducted. The strain-stress curve of the P-PDBA $100 \%$ sample that had healed for 3 days showed almost quantitative recovery of the mechanical properties as compared to the original uncut sample (Fig. 3b). The efficiency of healing, which was quantified as the percent recovery of toughness, was 91\% (Fig. 3c). As a control test excluding the water effect in self- healing, some cut P-PDBA 100\% samples were rejoined without exposure to seawater and kept in a desiccator $(<20 \%$ relative humidity) for 3 days. As shown in Table S2, $\uparrow$ the healing efficiency for these samples was less than $2 \%$.

\section{Water stability studies of network polymers}

Swelling tests under seawater. The water stability of P-PDBA $100 \%$ and $\mathrm{P}^{-\mathrm{Ca}^{2+}} 100 \%$ in seawater was also examined through the estimation of the swelling ratio and water contact angle. For swelling tests, P-PDBA $100 \%$ and $\mathrm{P}-\mathrm{Ca}^{2+} 100 \%$ were completely immersed into seawater, and the weight change was measured regularly until no further change in weight was observed. As shown in Fig. 4a, the P-PDBA 100\% film reached an equilibrium after 2 days and was swollen at most up to $2 \mathrm{wt} \%$, whereas P$\mathrm{Ca}^{2+} 100 \%$ was swollen up to $\sim 20 \mathrm{wt} \%$.

Water contact angle tests. To measure the water contact angle, a droplet of deionized water $(1 \mu \mathrm{L})$ was dropped onto the surfaces of the films, and the contact angles were measured (Fig. 4b). The contact angle of P-PDBA $100 \%$ was $103^{\circ}$, revealing the hydrophobicity of its surface. In comparison, the contact angles of $\mathrm{P}^{-\mathrm{Ca}^{2+}} 100 \%$ and non-crosslinked $\mathrm{P}(\mathrm{DA}-\mathrm{co}-\mathrm{BA})$ were $88^{\circ}$ and $103^{\circ}$, respectively. Hence, the hydrophobicity of P-PDBA $100 \%$ was no different from $\mathrm{P}(\mathrm{DA}-c o-\mathrm{BA})$, while $\mathrm{P}^{-\mathrm{Ca}^{2+}} 100 \%$ was found to be less hydrophobic. The swelling tests and water

Table 1 Summary of mechanical and thermal properties of polymers

\begin{tabular}{|c|c|c|c|c|c|}
\hline Sample & Young's modulus ${ }^{a}[\mathrm{MPa}]$ & Break strength [MPa] & Strain at break $\left[\mathrm{mm} \mathrm{mm}^{-1}\right]$ & Toughness $^{b}\left[\mathrm{MJ} \mathrm{m}^{-3}\right]$ & $T_{\mathrm{g}}\left[{ }^{\circ} \mathrm{C}\right]$ \\
\hline P-PDBA ${ }^{c} 100 \%$ & $2.3 \pm 0.3$ & $2.8 \pm 0.2$ & $5.1 \pm 0.4$ & $10.6 \pm 1.4$ & -11.8 \\
\hline P-PDBA $80 \%$ & $1.3 \pm 0.2$ & $2.2 \pm 0.2$ & $11.1 \pm 1.5$ & $10.0 \pm 0.4$ & -13.5 \\
\hline P-PDBA $60 \%$ & $0.2 \pm 0.03$ & $1.2 \pm 0.04$ & $13.9 \pm 3.7$ & $9.2 \pm 1.7$ & -17.3 \\
\hline $\mathrm{P}^{-\mathrm{Ca}^{2+}} 100 \%$ & $0.6 \pm 0.01$ & $2.3 \pm 0.1$ & $4.5 \pm 0.5$ & $5.4 \pm 0.8$ & -14.1 \\
\hline $\mathrm{P}(\mathrm{DA}-c o-\mathrm{BA})$ & $-^{d}$ & $-d$ & $-d$ & $-d$ & -19.3 \\
\hline
\end{tabular}

${ }^{a}$ Calculated from the initial slope of stress strain curves. ${ }^{b}$ Integration of the area under the stress-strain curves. ${ }^{c} 100 \%$ indicates $100 \%$ coordination feed ratio. ${ }^{d}$ Unable to measure because it is too soft. 
a)

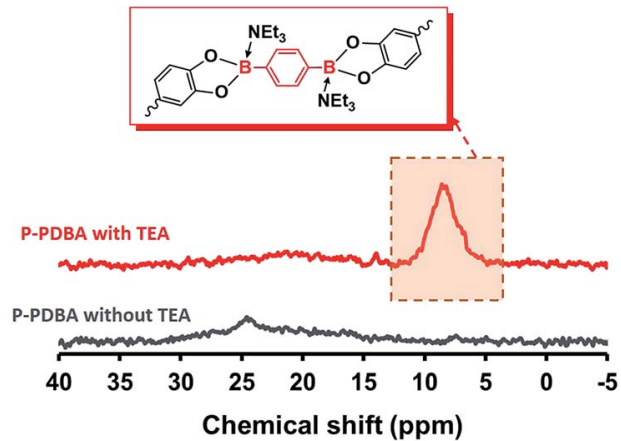

b)

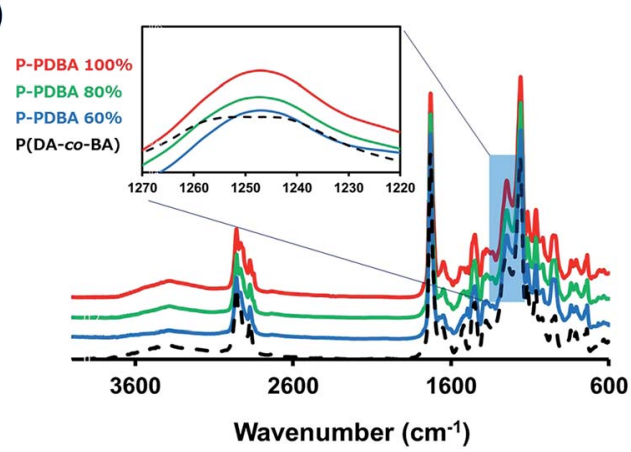

Fig. 2 (a) Solid-state ${ }^{11}$ B NMR of P-PDBA $100 \%$ with and without TEA. (b) ATR-FTIR spectra of P-PDBA polymers containing different amounts of the cross-linker (PDBA).

contact angle measurements indicate that P-PDBA 100\% possesses water stability with remarkably lower water absorption and higher hydrophobicity than $\mathrm{P}^{-\mathrm{Ca}^{2+}} 100 \%$, even though they are made from the same polymer; thus the high water a)

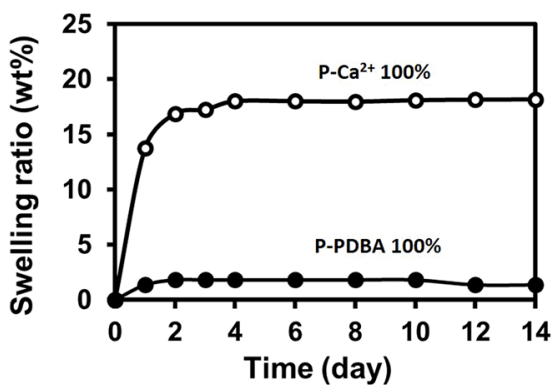

b)

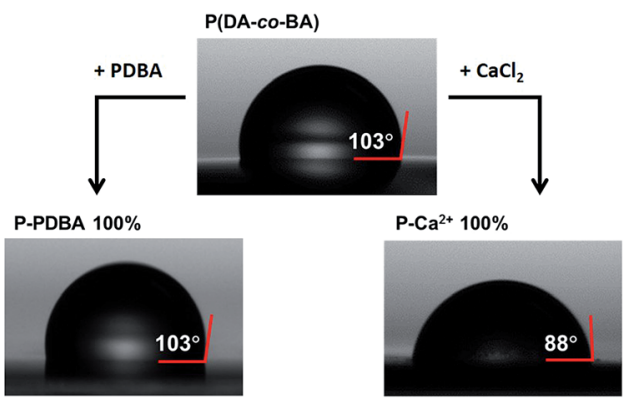

Fig. 4 (a) The swelling ratio of P-PDBA $100 \%$ and $\mathrm{P}-\mathrm{Ca}^{2+} 100 \%$ in seawater over a 2 week period. (b) Water contact angles of P(DA-coBA), P-PDBA $100 \%$, and $\mathrm{P}-\mathrm{Ca}^{2+} 100 \%$.

stability of P-PDBA $100 \%$ cannot be fully explained by the hydrophobicity of the polymer chain.

\section{Morphological characterizations of network polymers}

To study the origin of the superior water stability of P-PDBA $100 \%$ and the different water-resistivity of the two similar

a)
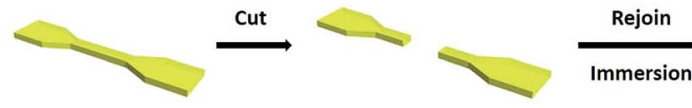

Submerged in seawater

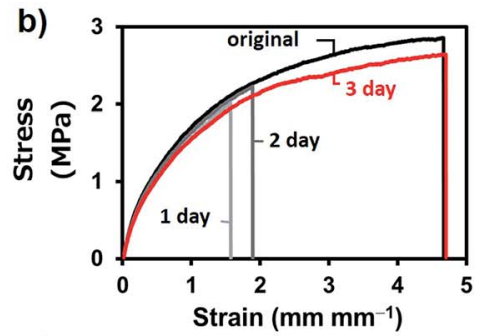

c)

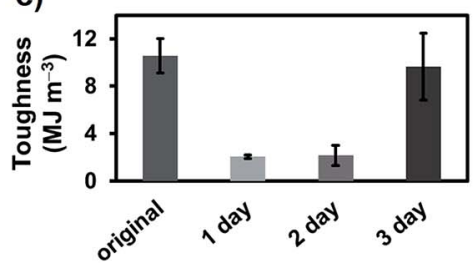

d)
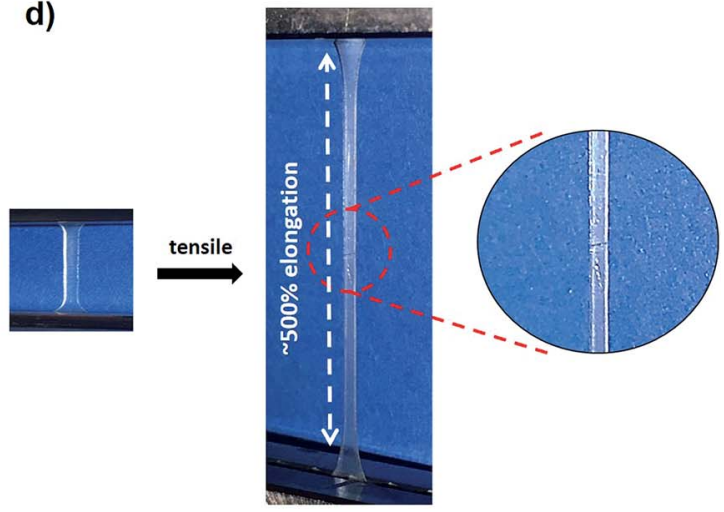

Fig. 3 (a) The self-healing test protocol. (b) Tensile stress-strain curves of original and healed polymers (1-3 days). (c) Toughness of the original and healed (for 1-3 days) samples. (d) Photographs of the tensile test of P-PDBA $100 \%$ after 3 days of healing in seawater. 
polymers, their mesoscopic morphologies were visualized by atomic-force microscopy (AFM, Fig. 5a and c) and transmission electron microscopy (TEM, Fig. 5b and d). Interestingly, the TEM and AFM images clearly showed a microphase-separated morphology in the $\mathrm{P}^{-\mathrm{Ca}^{2+}} 100 \%$ thin films. As granular domains $(<100 \mathrm{~nm})$ appeared darker in the TEM images (Fig. 5d), these domains had higher electron density, presumably due to the concentrated $\mathrm{Ca}^{2+}$ aggregates in the polymer matrix. In contrast, a notable phase-separation was not observed in the images of P-PDBA $100 \%$. These results indicate that the PDBA crosslinkers were finely dispersed in the polymer matrix as crosslinks, and the hydrophobicity of the polymer backbone itself could be well-maintained in the P-PDBA $100 \%$ structure. On the contrary, the electrostatic interactions between ions (i.e. catechol- $\mathrm{Ca}^{2+}$ complexes and $\mathrm{Cl}^{-}$) in $\mathrm{P}-\mathrm{Ca}^{2+}$ $100 \%$ could generate metal-concentrated domains (ion clusters) where the water uptake likely takes place due to the high dielectric constants, even though the polymer chain possessed hydrophobic properties (Fig. 5f). ${ }^{28,29}$ In the case of P-PDBA $100 \%$, covalently cross-linked PDBAs form sterically hindered tetrahedral boronate ester moieties with fully occupied $\mathrm{sp}^{3}$ orbitals; thus, no strong interactions could be formed between crosslink points in the solid state. Therefore, the PDBA crosslinkers were finely dispersed into the local hydrophobic environment, leading to highly suppressed water absorption (Fig. 5e).

\section{Stability of mechanical properties under seawater}

The stability of the mechanical properties of network polymers under seawater was examined. First, dumbbell-shaped films were stored in seawater for 3 days then tensile tests were conducted without drying process. As shown in Table S4, $\dagger$ even though Young's modulus and break strength were decreased when compared with their original properties, the strain at break of P-PDBA $100 \%$ was not changed with relatively high

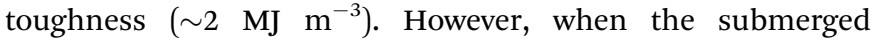
samples were dried before tensile tests, only P-PDBA 100\% showed nearly recovered mechanical properties. For further checking the under seawater stability of network polymers, 1 month storage tests were also carried out with drying process. Remarkably, as shown in Fig. 6a, the mechanical properties were completely unchanged (Young's modulus, break strength, and strain at break; see Table S5†), and its toughness was fully maintained ( $\sim 100 \%$ of original sample). In contrast, the $\mathrm{P}-\mathrm{Ca}^{2+}$ $100 \%$ showed deteriorated mechanical properties (increased Young's modulus but significantly decreased strain at break) in

a)

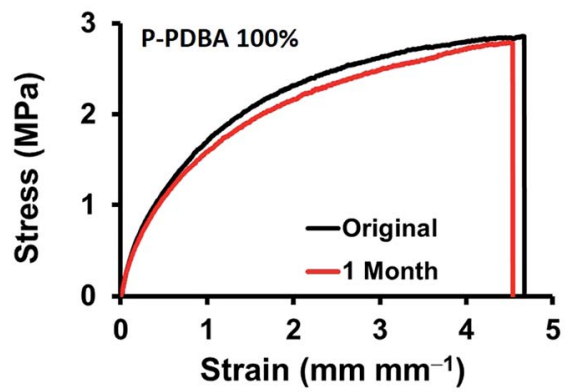

b)

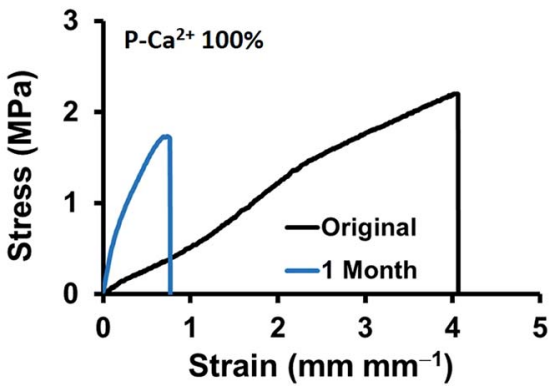

Fig. 6 Long-term stability under seawater. Tensile stress-strain curves of (a) P-PDBA $100 \%$, and (b) P-Ca ${ }^{2+}$ films incubated in seawater for 1 month. a)

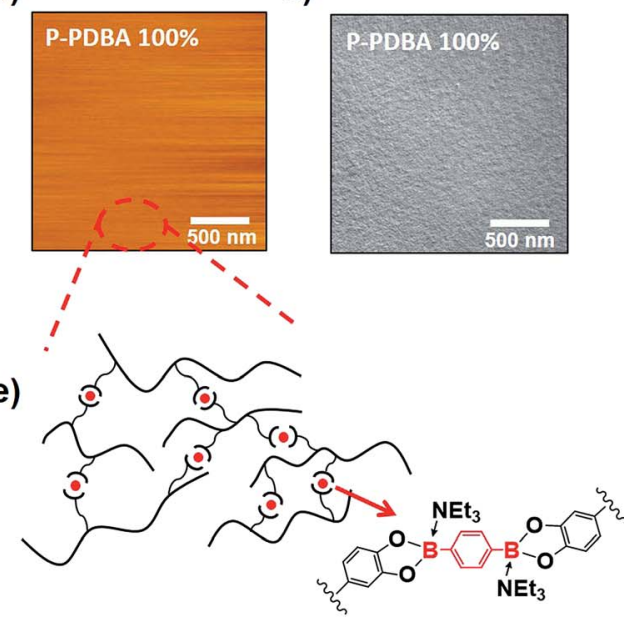

c)

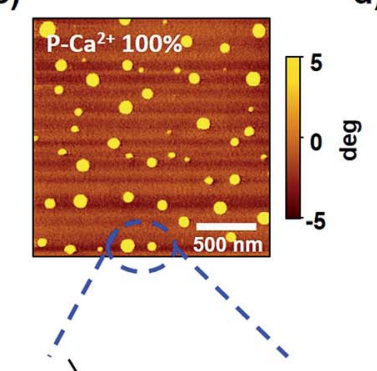

d)

f)

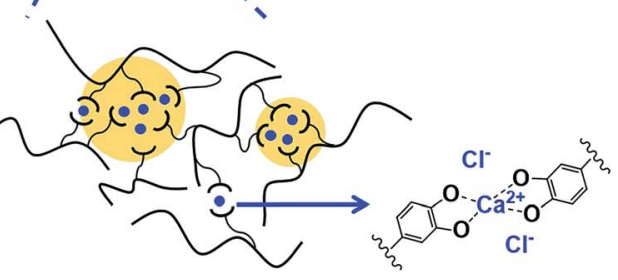

Fig. 5 (a) AFM phase images of P-PDBA $100 \%$. (b) TEM images of P-PDBA $100 \%$. (c) AFM phase images of P-Ca ${ }^{2+} 100 \%$. (d) TEM images of P-Ca ${ }^{2+}$ $100 \%$. (e) Covalently crosslinked P-PDBA $100 \%$ with finely dispersed crosslinkers. (f) lonically crosslinked P-Ca ${ }^{2+} 100 \%$ with aggregation of crosslinkers (ion clusters). 


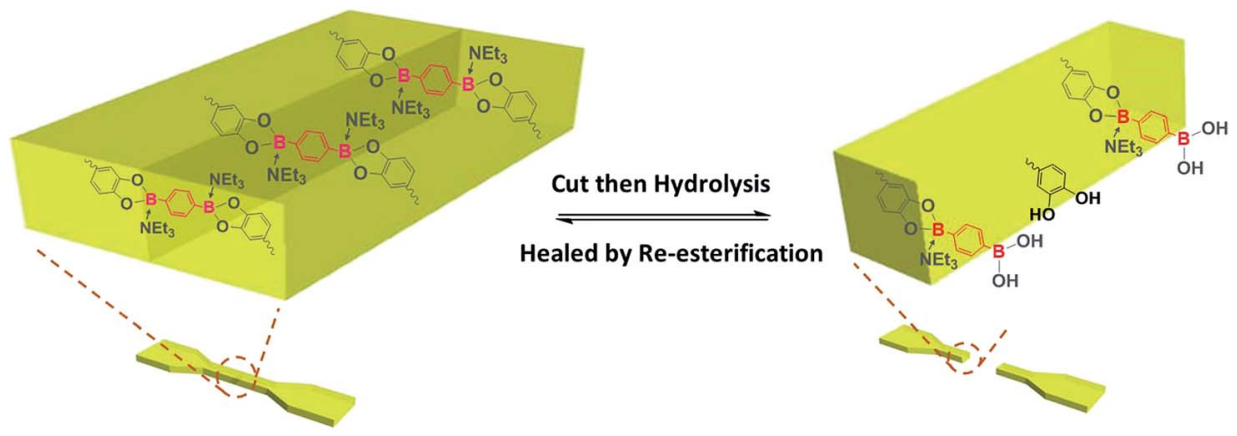

Fig. 7 Illustration of the water-triggered self-healing process.

the storage under seawater (Fig. 6b). These results indicate that the network changes of P-PDBA $100 \%$ (e.g. degradation or oxidation of polymer matrix) are highly suppressed under seawater conditions and even though mechanical properties can be affected by the hydrolysis of boron-catechol crosslinks through marginal water absorption $(\sim 2 \mathrm{wt} \%)$, they can be fully recovered with dry process.

\section{Self-healing mechanism without water absorption}

Taken together, the shape and mechanical properties of P-PDBA $100 \%$ were well-maintained in seawater because of the suppressed water absorption. The surface of P-PDBA 100\%, however, slightly swells with marginal water absorption, which is the key factor for its successful self-healing. The mean surface roughness $\left(R_{\mathrm{a}}\right)$ of P-PDBA $100 \%$ film was increased after incubation in seawater for three days (Fig. S4 $\dagger$ ). A possible mechanism for healing behavior is illustrated in Fig. 7. Absorbed water enhances the dynamics of esterification between the catechol and boronic acid moieties and shifts the equilibrium of the reactions slightly toward hydrolysis, resulting in the exposure of free catechol and boronic acid groups on the surface. By attaching the cut surfaces, the functional groups are allowed to form the boronate ester bonds again by esterification. This healing mechanism only works at the outermost surfaces of the crack, where the polymer is water-swollen.

\section{Conclusions}

In summary, we designed and synthesized tough, self-healing, and non-swellable materials from a catechol-based polymer crosslinked with PDBA. P-PDBA 100\% showed good stability and remarkable self-healing ability under seawater. Moreover, by comparing with a metal-catechol based polymer $\left(\mathrm{P}^{-C^{2+}}\right.$ $100 \%$ ), it was proven that the boron-containing catechol polymer has superior self-healing abilities, stability, and transparency under seawater. Water stability and morphology studies revealed that the water affinity of the catechol-based polymers is affected not only by the hydrophobicity of polymer chain, but also by their mesoscopic morphologies which can be controlled by judiciously selecting crosslinkers. We envisage that the design concept of the water-assisted selfhealable P-PDBA $100 \%$ with high water stability provides a valuable starting point for further development of self-healing polymeric materials used in diverse wet conditions.

\section{Acknowledgements}

This work was financially supported by JSPS KAKENHI Grant No. 15 K17440.

\section{Notes and references}

1 M. Doble, R. Venkatesan and N. V. R. Kumar, Polymers in a Marine Environment, Smithers, 2014.

2 P. Le Gac, P. Davies and D. Choqueuse, Oil Gas Sci. Technol., 2015, 70, 279-289.

3 A. Akthakul, R. F. Salinaro and A. M. Mayes, Macromolecules, 2004, 37, 7663-7668.

4 R. T. Martin, L. P. Camargo and S. A. Miller, Green Chem., 2014, 16, 1768-1773.

5 X. Chen, M. A. Dam, K. Ono, A. Mal, H. Shen, S. R. Nutt, K. Sheran and F. Wudl, Science, 2002, 295, 1698-1702.

6 P. Cordier, F. Tournilhac, C. Soulié-Ziakovic and L. Leibler, Nature, 2008, 451, 977-980.

7 Y. Chen, A. M. Kushner, G. A. Williams and Z. Guan, Nat. Chem., 2012, 4, 467-472.

8 S. Burattini, B. W. Greenland, D. H. Merino, W. Weng, J. Seppala, H. M. Colquhoun, W. Hayes, M. E. Mackay, I. W. Hamley and S. J. Rowan, J. Am. Chem. Soc., 2010, 132, 12051-12058.

9 H. Ying, Y. Zhang and J. Cheng, Nat. Commun., 2014, 5, 32183226.

10 V. K. Thakur and M. R. Kessler, Polymer, 2015, 69, 369-383. 11 B. K. Ahn, D. W. Lee, J. N. Israelachvili and J. H. Waite, Nat. Mater., 2014, 13, 867-872.

12 J. J. Cash, T. Kubo, A. P. Bapat and B. S. Sumerlin, Macromolecules, 2015, 48, 2098-2106.

$13 \mathrm{~J} . \mathrm{Li}, \mathrm{H}$. Ejima and N. Yoshie, ACS Appl. Mater. Interfaces, 2016, 8, 19047-19053.

14 N. N. Xia, X. M. Xiong, J. Wang, M. Z. Rong and M. Q. Zhang, Chem. Sci., 2016, 7, 2736-2742.

15 H. Kamata, K. Kushiro, M. Takai, U. Chung and T. Sakai, Angew. Chem., Int. Ed., 2016, 55, 9282-9286.

16 K.-L. G. Ho, A. L. Pometto and P. N. Hinz, J. Environ. Polym. Degrad., 1999, 7, 83-92. 
17 J. H. Waite, Int. J. Adhes. Adhes., 1987, 7, 9-14.

18 H. Shao and R. J. Stewart, Adv. Mater., 2010, 22, 729-733.

19 N. Holten-Andersen, M. J. Harrington, H. Birkedal, B. P. Lee, P. B. Messersmith, K. Y. C. Lee and J. H. Waite, Proc. Natl. Acad. Sci. U. S. A., 2011, 108, 2651-2655.

20 M. Krogsgaard, M. A. Behrens, J. S. Pedersen and H. Birkedal, Biomacromolecules, 2013, 14, 297-301.

21 M. J. Harrington, A. Masic, N. Holten-Andersen, J. H. Waite and P. Fratzl, Science, 2010, 328, 216-220.

22 L. He, D. E. Fullenkamp, J. G. Rivera and P. B. Messersmith, Chem. Commun., 2011, 47, 7497-7499.

23 R. Nishiyabu, Y. Kubo, T. D. James and J. S. Fossey, Chem. Commun., 2011, 47, 1124-1150.
24 S. L. Diemer, M. Kristensen, B. Rasmussen, S. R. Beeren and M. Pittelkow, Int. J. Mol. Sci., 2015, 16, 21858-21872.

25 R. p. Singhal, B. Ramamurhy, N. Govindraj and Y. Sarwar, J. Chromatogr. A, 1991, 543, 17-38.

26 N. Luisier, R. Scopelliti and K. Severin, Soft Matter, 2016, 12, 588-593.

27 I. A. Janković, Z. V. Šaponjić, E. S. Džunuzović and J. M. Nedeljković, Nanoscale Res. Lett., 2009, 5, 81-88.

28 A. Eisenberg and M. Navratil, Macromolecules, 1974, 7, 9094.

29 S. R. Lowry and K. A. Mauritz, J. Am. Chem. Soc., 1980, 102, 4665-4667. 